\title{
FACTORISATION OF CHARACTERISTIC FUNCTIONS ON NONCOMMUTATIVE GROUPS
}

\author{
AUBREY WULFSOHN
}

\begin{abstract}
A characteristic function, without idempotent factors, on a separable compact group is decomposed, modulo characters, as a product of indecomposable characteristic functions and an infinitely divisible characteristic function.
\end{abstract}

A continuous normalized positive definite function on a topological group $G$ will be called a characteristic function. Denote by $|\phi|^{2}$ the characteristic function defined by $|\phi|^{2}(g)=|\phi(g)|^{2}$ for all $g$ in $G$. The characteristic function identically 1 will be called degenerate. A continuous homomorphism of $G$ to $\mathbf{C}^{*}$, the group of complex numbers modulo 1 , will be called a character. We are concerned with the factorisation of a characteristic function as a product of characteristic functions where we write $\phi=\phi_{1} \phi_{2}$ if $\phi(g)=\phi_{1}(g) \phi_{2}(g)$ for all $g$ in $G$. A characteristic function $\phi$ is called indecomposable if it cannot be expressed as a product of two other characteristic functions, idempotent if $\phi=\phi^{2}$ and infinitely divisible if for each $n \in \mathbf{N}$ one may write $\phi=\prod_{i=1}^{n} \phi_{i}^{(n)}$ for some characteristic function $\phi^{(n)}$, each $\phi_{i}^{(n)}=\phi^{(n)}$. Denote the set of factors of $\phi$ by $F_{\phi}$, the set of indecomposable factors of $\phi$ by $I F_{\phi}$ and the subgroup of $G$ generated by $\{g: \phi(g) \neq 0\}$ by $G_{\phi}$. Denote left Haar measure on a separable locally compact group by $d g$.

For the purposes of factorisation we shall consider two characteristic functions $\phi_{1}$ and $\phi_{2}$ to be equivalent if $\phi_{1}=\phi_{2} \chi$ where $\chi$ is a character. When $G$ is commutative a characteristic function is the Fourier transform of a probability measure on the dual group $\hat{G}$ and equivalent characteristic functions are the Fourier transforms of shift-equivalent measures on $\hat{G}[4]$.

A. I. Khinchin [2] showed that the characteristic function of a probability distribution on $\mathbf{R}$ can be represented as $\phi_{2} \phi_{3}$ where $\phi_{2}$ is a denumerable product of indecomposable factors, $\phi_{3}$ has no indecomposable factors and is necessarily infinitely divisible. K. R. Parthasarathy, R. Ranga Rao and S. R. S. Varadhan [3] extended this.result to a characteristic function on an arbitrary separable locally compact commutative group decomposing it as $\phi_{1} \phi_{2} \phi_{3}$ where $\phi_{1}$ is idempotent, $\phi_{2}$ and $\phi_{3}$ as above. When the group has no compact subgroups there is no proper idempotent factor.

Received by the editors December 22, 1981.

1980 Mathematics Subject Classification. Primary 46L50, 43A30; Secondary 22C05.

Key words and phrases. Characteristic function, compact separable group, idempotent factor, indecomposable factor, infinitely divisible factor. 
The factorisation can be translated to the positive-definite matrices $\left[\alpha_{i j}\right]=$ [ $\left.\phi\left(g_{i} g_{j}^{-1}\right)\right]$ for sequences $\left(g_{i}\right)$ in $G$. The product of characteristic functions corresponds to coefficientwise multiplication of the matrices, and matrices $\left[\alpha_{i j}\right]$ and $\left[\beta_{i j}\right]$ correspond to equivalent characteristic functions if and only if $\alpha_{i j}=\beta_{i j} c_{i} c_{j}$ for $c_{i}, c_{j} \in \mathbf{C}^{*}$.

In $\$ 1$ we consider the cancellation of idempotent factors from a characteristic function on a topological group and find conditions determining whether a characteristic function has idempotent factors or not. In $\$ 2$ we prove Khinchin's factorisation theorem for a characteristic function, without idempotent factors, on a separable compact group. We have not been able to prove Khinchin's theorem for characteristic functions with idempotent factors, neither have we been able to construct a counterexample. In $\$ 3$ we show why, in the commutative case, any characteristic function can be factorised as above.

\section{Idempotent factors of a characteristic function on a topological group.}

Proposition 1. Let $G$ be a topological group. If $\psi$ is an idempotent factor of a characteristic function $\phi$ of $G$ then $\psi=\chi_{H}$ where $H$ is an open and closed subgroup of $G$. The maximal idempotent factor, i.e. that with the minimal support and so the least degenerate, is $\chi_{G_{\phi}}$. One factorises $\phi$ as $\chi_{G_{\phi}} \phi_{0}$ where $\phi_{0}$ is the restriction of $\phi$ to $G_{\phi}$.

Proof. An idempotent is necessarily of the form $\chi_{H}$ for a subset $H$ of $G$. Since the factors are required to be continuous it follows that $H$ is open and closed and, since $\psi\left(g_{1}\right)=1, \psi\left(g_{2}\right)=1$ implies $\psi\left(g_{1} g_{2}\right)=1,[1,32.7]$, it follows that $H$ must be an open (and closed) subgroup. For $\chi_{H}$ to be a factor of $\phi$, necessarily $\chi_{H}(g) \neq 0$ whenever $\phi(g) \neq 0$, so $H \supset G_{\phi}$. By construction $G_{\phi}$ is an open subgroup and so also closed. By [1, 32.43], $\phi_{0}$ is also a characteristic function for $G$.

COROLLARY 1. The characteristic function $\phi$ has nondegenerate idempotent factors if and only if $G_{\phi} \neq G$.

Proposition 2. Let $\phi$ be an infinitely divisible characteristic function on a group $G$. It has a nondegenerate idempotent factor if and only if it has zeros.

Proof. The function $\phi_{1}=\lim _{n}|\phi|^{2^{-n}}$ is the idempotent factor, where $|\phi|(g)=$ $|\phi(g)|$ for all $g$ in $G$. Indeed, $\phi \bar{\phi}$ is a characteristic function and, since $\phi$ is infinitely divisible, its repeated square roots will exist and be characteristic functions, Furthermore $\phi_{1}=\chi_{G_{\phi}}$.

This generalises Lemma 4.2 of [5], proved there for compact $G$.

2. Characteristic functions, without idempotent factors, on a separable compact group.

LEMMA 1. Let $G$ be a separable compact group and $\left(\phi_{n}\right)$ a sequence of characteristic functions such that $\int_{G}\left|\phi_{n}(g)\right|^{2} d g \rightarrow 1$ as $n \rightarrow \infty$. Then there exists a sequence $\left(\chi_{n}\right)$ of characters such that $\phi_{n} \chi_{n}$ converges uniformly to the degenerate characteristic function as $n \rightarrow \infty$. 
Proof. The proof is contained explicitly in the proof of [5, Lemma 4.1].

For a characteristic function $\phi$, without idempotent factors, on a compact group we define the Khinchin functional $N_{\phi}$ on $F_{\phi}$, which measures 'departure' from the degenerate characteristic functional, by $N_{\phi}(\psi)=-\int_{G} \log |\psi(g)| d g$. It is well defined and convergent since $G$ is generated by a sequence of elements $\left(g_{i}\right)$ such that $\phi\left(g_{i}\right) \neq 0$ for all $i$, and since $G$ is compact, $N_{\phi}$ is bounded.

Proposition 3. Let $\phi$ be a characteristic functional without idempotent factors on a separable compact group $G$. If $\psi_{1}, \psi_{2}, \psi \in F_{\phi}$

(i) $N_{\phi}\left(\psi_{1} \psi_{2}\right)=N_{\phi}\left(\psi_{1}\right)+N_{\phi}\left(\psi_{2}\right)$,

(ii) $N_{\phi}(\psi) \geqslant \int_{G}(1-|\psi(g)|) d g \geqslant 0$,

(iii) $N_{\phi}(\psi)=0$ if and only if $\psi$ is equivalent to the degenerate characteristic function.

Proof. Properties (i) and (ii) are obvious. Property (iii) follows since $N_{\phi}\left(\chi_{G}\right)=0$ and $N_{\phi}(\chi)=0$ for any character $\chi$; if $N_{\phi}(\psi)=0$ then

$$
\int_{G}\left(1-|\psi(g)|^{2}\right) d g \leqslant 2 \int_{G}(1-|\psi(g)|) d g=0
$$

by (ii), and, by Lemma 1 , there exists a character $\chi$ such that $\psi \chi$ is degenerate.

LEMMA 2. Let $\phi$ be a characteristic function without idempotent factors on a separable compact group $G$ and let $\left(\psi_{i}\right)$ be a sequence of factors of $\phi$ such that for all $n \in \mathbf{N}$ the product $\prod_{i=1}^{n} \psi_{i}$ is also a factor of $\phi$. Then there exist characters $\chi_{i}$ such that $\prod_{i=1}^{n} \psi_{i} \chi_{i}$ converges to a characteristic function as $n \rightarrow \infty$.

Proof. $\sum N_{\phi}\left(\psi_{i}\right) \leqslant N_{\phi}(\phi)$ so $\sum_{i=k}^{\infty} N_{\phi}\left(\psi_{i}\right)$ and $N_{\phi}\left(\prod_{i=k}^{\infty} \psi_{i}\right)$ converge to zero as $k \rightarrow \infty$. By Lemma 1 there exist $\left(\chi_{k}\right)$ such that $\left(\chi_{k-1} \prod_{i=k}^{\infty} \psi_{i}\right)$ converges to the degenerate characteristic function as $k \rightarrow \infty$. Thus, absorbing each $\chi_{k-1}$ in the preceding finite product, $\prod_{i=1}^{n} \psi_{i} \chi_{i}$ converges to a characteristic function as $n \rightarrow \infty$.

Proposition 4. Let $\phi$ be a real-valued characteristic function on a compact group. Every sequence in $F_{\phi}$ has a convergent subsequence.

Proof. The set $F_{\phi}$ is equicontinuous since, for $\psi \in F_{\phi}$,

$$
|\psi(g)-\psi(h)|^{2} \leqslant 2\left(1-\operatorname{Re} \psi\left(g^{-1} h\right)\right) \leqslant 2\left(1-\phi\left(g^{-1} h\right)\right) .
$$

The proposition follows from the Arzela-Ascoli theorem.

Lemma 2 is the noncommutative version of [4, Theorem III.5.3], Proposition 4 is an analogue of Corollary III.5.2.

Proposition 5. Let $G$ be a separable compact group. Any characteristic function without idempotent factors can be factorized, modulo a character, as a product of a denumerable number of indecomposable characteristic functions and a characteristic function with no indecomposable factors.

Proof. If $\phi$ does not have any indecomposable factors the proposition holds. Suppose $\phi$ has indecomposable factors. Write $\operatorname{Sup}\left\{N_{\phi}(\psi): \psi \in I F_{\phi}\right\}=\delta(\phi)$. One can decompose $\phi$ as $\psi_{1} \lambda_{1}$ where $N_{\phi}\left(\psi_{1}\right) \geqslant \frac{1}{2} \delta(\phi)$ and decompose the characteristic function $\lambda_{n-1}$ as $\psi_{n} \lambda_{n}$ where $N_{\phi}\left(\psi_{n}\right) \geqslant \frac{1}{2} \delta\left(\lambda_{n-1}\right)$, for $n=2,3, \ldots$ If $\lambda_{k}$ has no 
indecomposable factors for some $k$ the process terminates and the proposition holds. When the process does not terminate there exist, by Lemma 2, characters $\chi_{i}$ such that $\Pi \psi_{i} \chi_{i}$ converges. So $N_{\phi}\left(\psi_{n}\right) \rightarrow 0$ as $n \rightarrow \infty$. So also $\lambda_{n}$ will converge to a characteristic function $\lambda$ as $n \rightarrow \infty$. If $\lambda$ has an indecomposable factor $\psi$ then $\psi \in F_{\lambda_{n}}$ for all $n$ and so $N_{\phi}(\psi) \leqslant \delta\left(\lambda_{n}\right)$ for all $n$; as $\delta\left(\lambda_{n}\right) \leqslant 2 N_{\phi}\left(\psi_{n+1}\right) \rightarrow 0$ as $n \rightarrow \infty$, it follows from Proposition 3 that $\psi$ is a character.

LEMMA 3. Let $\phi$ be a characteristic function, with no indecomposable factors and with no idempotent factors, on a separable compact group $G$. There exists a sequence of decompositions $\left(D_{n}\right)$ of $\psi$ such that $\nu=\inf _{n} \sup \left\{1-|\psi(g)|: \psi \in D_{n}, g \in G\right\}=0$.

Proof. For any decomposition $D$ of $\phi$ let

$$
\nu_{D}=\sup \{1-|\psi(g)|: \psi \in D, g \in G\} .
$$

For any characteristic function $\tau$, if $\psi \in F_{\tau}$ then $1-|\psi(g)| \leqslant 1-|\tau(g)|$ for all $g$ in $G$. One can arrange an array of decompositions

$$
\left(D_{n}: \phi=\phi_{n, 1} \cdots \phi_{n, k_{n}}\right)
$$

such that $\nu_{D_{n}} \rightarrow \nu$ as $n \rightarrow \infty, 1-\left|\phi_{n, j}(g)\right| \leqslant 1-\left|\phi_{n, 1}(g)\right|$ for all $g \in G, 1<j \leqslant k_{n}$, and $1-\left|\phi_{n, 1}(g)\right|=\nu_{D_{n}}$ for some $g$. Using Lemma 2, $\phi$ can be decomposed as $\phi_{1} \phi_{2}$, where $\phi_{2}=\lim _{n} \prod_{j=2}^{k_{n}} \chi_{n, j} \phi_{n, j}$, for an array $\left(\chi_{n, i}\right)$ of characters of $G$, and such that $1-\left|\phi_{1}(g)\right|=\nu$ for some $g$. Since $\phi_{1}$ and $\phi_{2}$ are again decomposable $\nu$ must be 0 .

An array of decompositions $\left(D_{n}\right)$ such that $\nu=0$ will be called uniformly infinitesimal.

COROLlARY 2. If a characteristic function $\phi$ on a compact separable group has neither idempotent nor indecomposable factors then $\{g: \phi(g) \neq 0\}=G_{\phi}$.

Proof. Since $G_{\phi}=G_{|\phi|^{2}}$ it is sufficient to prove that if $|\phi|^{2}\left(g_{1}\right)>0$ and $|\phi|^{2}\left(g_{2}\right)$ $>0$ then $|\phi|^{2}\left(g_{1} g_{2}\right)>0$. Choose a uniformly infinitesimal array of decompositions $\left(\phi_{n, 1} \cdots \phi_{n, k_{n}}\right)_{n}$ of $\phi$. For each of the decompositions $|\phi|^{2}(g)=\left|\phi_{n, 1}\right|^{2}(g) \cdots$ $\left|\phi_{n, k_{n}}\right|^{2}(g)$. Thus $|\phi|^{2}(g)>0$ if and only if, for any $n,\left|\phi_{n, j}\right|^{2}(g)>0,1 \leqslant j \leqslant k_{n}$, and so also if and only if $\lim _{n}\left(n-\left|\phi_{n, j}\right|^{2}(g)\right)<\infty$ for $j \in \mathbf{N}$. The corollary follows using [5, Lemma 3.6].

Proposition 6. A characteristic function $\phi$, with neither idempotent nor indecomposable factors, on a compact separable group $G$, is, modulo a character, infinitely divisible.

Proof. By Corollary $1, G=G_{\phi}$. We denote $\phi\left(h^{-1} g\right)\left(\phi\left(h^{-1}\right) \phi(g)\right)^{-1}$ by $K(g, h)$, adding suffixes if required. By Lemma 3 we can find a uniformly infinitesimal array $\left(\phi_{n, 1} \cdots \phi_{n, k_{n}}\right)_{n}$ of decompositions of $\phi$ such that, for large enough $n, 1-\left|\phi_{n, j}(g)\right|$ is as small as we like. By [6, Lemma 3.5],

$$
\left|K_{n, j}(g, h)\right| \leqslant 2\left(1-\left|\phi_{n, j}\left(h^{-1}\right)\right|\right)^{1 / 2}\left(1-\left|\phi_{n, j}(g)\right|\right)^{1 / 2}\left(\phi_{n, j}\left(h^{-1}\right) \phi_{n, j}(g)\right)^{-1}
$$

for $n \in \mathbf{N}, 1 \leqslant j \leqslant k_{n}$. So $\lim _{n} \sup _{j}\left|1-K_{n, j}(g, h)\right|=0$. Using the procedure of [6, Lemma 4.2] we can define $L(g, h)=\log K(g, h)$ and prove it to be continuous and positive-definite on $G \times G$. As in [6, Lemma 4.3], $L\left(h, g^{-1}\right)$ is an additive 2-cocycle. 
It is a coboundary since $H_{2}(G, \mathbf{R})=\{0\}$ and the real and imaginary parts of $L$ can be considered separately. Hence $L(g, h)=\psi\left(h^{-1} g\right)-\psi\left(h^{-1}\right)-\psi(g)$ for some continuous conditionally positive-definite function $\psi$ on $G$. By [5, Theorem 4.1], $e^{\psi}$ is infinitely divisible. As in the proof of [6, Theorem 5.1], $e^{\psi}=\phi \chi$ for some character $\chi$ of $G$.

COROLlARY 3. On a separable compact group, if a characteristic function has no idempotent factors then it has indecomposable factors whenever it has zeros.

Proof. Suppose $\phi$ has zeros but no indecomposable factors. By Proposition 6 it is infinitely divisible so by Proposition 2 it cannot have zeros.

THEOREM. Let $G$ be a separable compact group and $\phi$ a characteristic function on $G$ with no idempotent factors. Then $\phi$ can be decomposed, modulo a character, as a product of indecomposable characteristic functions and an infinitely divisible characteristic function.

Proof. The theorem follows from Propositions 5 and 6.

3. Commutative groups. The method in [3] for proving Lemma 2 for a locally compact separable commutative group is to use [4, Corollary III.5.2], the analogue of our Proposition 4, to prove the existence of characters $\chi_{i}$ such that products $\Pi_{i} \psi_{i} \chi_{i}$ converge, and [4, Theorem III.5.2] to prove that all such convergent products are equivalent. Lemma 5 is [4, Theorem III.5.2] with a simpler proof than the original.

LEMMA 4. Let $G$ be a complete separable metric commutative group. If $\phi$ and $\psi$ are characteristic functions on $G$ such that $\phi \psi$ is the degenerate characteristic function, then $\phi$ and $\psi$ are characters.

PROOF. Denote the measure corresponding to a characteristic function $\zeta$ by $\mu_{\zeta}$. Since $\mu_{\phi} * \mu_{\psi}$ is the unit mass at the neutral element of $G$, so $\mu_{\phi}$ and $\mu_{\psi}$ must be point masses. Hence $\phi$ and $\psi$ are characters.

LEMMA 5. Let $\phi$ and $\psi$ be characteristic functions on a complete separable metric commutative group. If $\phi \in F_{\psi}$ and $\psi \in F_{\phi}$, then $\phi$ is equivalent to $\psi$.

Proof. The lemma follows from Lemma 4. Indeed, if $\phi=\chi_{1} \psi$ and $\psi=\chi_{2} \phi$, then $\phi=\chi_{1} \chi_{2} \phi$, so $\chi_{1} \chi_{2}$ is degenerate.

LEMMA 6. Let $\phi$ be a characteristic function on a locally compact separable commutative group $G$. Any character on $G_{\phi}$ extends uniquely to a character of $G$.

Proof. Denote the annihilator of $G_{\phi}$ in $\hat{G}$ by $K$ and identify $\hat{G}_{\phi}=\hat{G} / K$ with a Borel section $B$ of $G$. As an element of $B$ is also an element of $G$, a character of $G_{\phi}$ uniquely determines a character of $G$.

Let $\phi$ be a characteristic function on a locally compact separable commutative group $G$. By Proposition 1, $\phi$ can be factorised as $\chi_{G_{\phi}} \phi_{0}$. Propositions 5 and 6 hold for $\phi_{0}$ on $G_{\phi}$ [3]. By Lemma 6 the characters of $G_{\phi}$ occurring in the factorisation extend to characters of $G$. Thus $\phi$ can be factorised as $\phi_{1} \phi_{2} \phi_{3}$ as stated in the introduction. 
The author wishes to thank Professor K. R. Parthasarathy for suggesting the problem and for his criticism of an earlier version.

\section{REFERENCES}

1. E. Hewitt and K. A. Ross, Abstract harmonic analysis, Springer-Verlag, Berlin and New York, 1970.

2. A. I. Khinchin, Contribution à l'arithmétique des lois de distribution, Bull. Univ. Etat Moscow 1 (1937), 6-17.

3. K. R. Parthasarathy, R. Ranga Rao and S. R. S. Varadhan, Probability distributions on locally compact abelian groups, Illinois J. Math. 7 (1963), 337-369.

4. K. R. Parthasarathy, Probability measures on metric spaces, Academic Press, New York, 1967.

5. Infinitely divisible representations and positive definite functions on a compact group, Comm. Math. Phys. 16 (1970), 148-156.

6. K. Schmidt, Limits of uniformly infinitesimal families of projective representations of locally compact groups, Math. Ann. 192 (1971), 107-118.

Mathematics faculty, Open University, Milton Keynes, MK7 6AA, Great Britain 